Pacific Journal of Mathematics

THE SEMICONTINUITY OF THE MOST GENERAL INTEGRAL
OF THE CALCULUS OF VARIATIONS IN NON-PARAMETRIC
FORM

LIONELLO LOMBARDI 


\section{THE SEMICONTINUITY OF THE MOST GENERAL INTEGRAL OF THE CALCULUS OF VARIATIONS IN NON-PARAMETRIC FORM.*}

\section{LIONELLO LOMBARDI}

Summary. The positive quasi-regularity of integrals depending upon any number of surfaces in non-parametric form, each with any number of dimensions, is defined. Positive quasi regularity is proved to be sufficient for lower semicontinuity.

1. Let $D_{i}(i=1,2, \cdots, m)$ be a closed bounded set of the $n$-dimensional euclidean space of the variable vector $x_{i} \equiv\left\{x_{i}^{j}\right\}(j=1,2, \cdots, n)$, bounded by surfaces which are absolutely continuous in the sense of Tonelli $[60,62,63]$, without multiple points, and let $D$ be the cartesian product $\prod_{1}^{m} D_{i}$. Let $y \equiv\left\{y_{i}\right\}(i=1,2, \cdots, m)$ denote a vertical $m$-vector, and let $p$ denote a mxn matrix, whose row-vectors are $p_{i} \equiv\left\{p_{i}^{j}\right\}(j=1,2, \cdots$, $n)$. Let $x$ be the $m \cdot n$ matrix whose row-vectors are $x_{i}$ and $\phi[x, y, p]$ a real function, defined for $x_{i} \in D_{i}(i=1,2, \cdots, m)$ and for any $y$ and $p$, which is continuous with all its partial derivatives of the types

$$
\frac{\partial \phi[x, y, p]}{\partial p_{r}^{s}}, \frac{\partial^{2} \phi[x, y, p]}{\partial p_{r}^{s} \partial p_{r}^{t}} \quad(r=1, \cdots, m ; s, t=1, \cdots n) .
$$

Let $q=m$ be a positive integer and let $U_{q}$ denote a set of distinct positive integers out of the first $m$; let $\zeta$ be an index ranging over $U_{q}$, and let $\mu(\delta)$ be a mapping of $U_{q}$ into the set of the first $n$ integers. It will be assumed throughout that, for every $q$, every $U_{q}$ and every $\mu(\zeta)$, all the partial derivatives

$$
\frac{\partial^{2 q} \phi[x, y, p]}{\prod_{1}^{q} \partial x_{\zeta}^{\mu(\zeta)} \partial p_{\zeta}^{\mu(\zeta)}}
$$

exist and are continuous for every $x \in D$ and for every $y$ and $p$.

Let $y(x) \equiv\left\{y_{i}\left(x_{i}\right)\right\}(i=1,2, \cdots, m)$ denote a vector-valued function of the matrix $x$, such that each component $y_{i}\left(x_{i}\right)$ depends only upon the row vector $x_{i}$. We assume that each $y_{i}\left(x_{i}\right)$ is absolutely continuous, in the sense of Tonelli [63]; we shall call Variety $V$ the set of $m$ surfaces represented by $y(x)$.

Received July 29, 1960. The preparation of this paper was sponsored in part by the Office of Naval Research and the Office of Ordnance Research, U. S. Army. Reproduction in whole or in part is permitted for any purpose of the United States Government. The author also wishes to acknowledge the help given Gerald W. Kimble in the preparation of this paper. The author is now with Scientific Information Treatment Centre, EURATOM. 
We shall say that $V$ is of class 1 if all the first partial derivatives of all the $y_{i}\left(x_{i}\right)$ exist and are continuous; we shall say the $V$ is of class 2 if the same is also true for all the partial derivatives of the second order.

Let

$$
p_{i}^{\jmath}(x) \equiv \frac{\partial y_{i}\left(x_{i}\right)}{\partial x_{i}^{j}} \quad(i=1,2, \cdots m ; j=1,2, \cdots, n),
$$

and

$$
d x \equiv \prod_{1}^{m} d x_{i} \equiv \prod_{1}^{m} \prod_{i}^{n}{ }_{j} d x_{i}^{j}
$$

The $m \cdot n$ integral'

$$
I_{V}=\int_{D} \phi[x, y(x), p(x)] d x
$$

is called variety integral in non-parametric form; all the varieties $V$ where $I$ exists and is finite are called ordinary.

REMARK 1.1. Varieties $V$ of class 1 and 2 are ordinary for any function $\phi[x, y, p]$.

Let $\bar{p} \equiv\left\{\bar{p}_{i}\right\} \equiv\left\{\bar{p}_{i}^{j}\right\}$ denote another variable in the space of the matrix $p, \bar{y} \equiv\left\{\bar{y}_{i}\right\}$ another variable in the space of the vector $y, V \equiv \bar{y}(x) \equiv\left\{\bar{y}_{i}\left(x_{i}\right)\right\}$ another variety $V$; let

$$
\bar{p}_{i}^{j}\left(x_{i}\right)=\frac{\partial \bar{y}_{i}\left(x_{i}\right)}{\partial x_{i}^{j}}
$$

the distance $\rho(V, \bar{V})$ between $V$ and $\bar{V}$ is defined by the formula

$$
\rho(V, \bar{V})=\sup _{x, i}\left|y_{i}(x)-\bar{y}_{i}(x)\right| \text {. }
$$

Continuity and semicontinuity of the real function $I_{V}$ will be considered throughout with respect to this m-uniform metric.

In one of our previous papers [33] the following theorem was proved:

Continuity THEOREM 1.2. Necessary and sufficient conditions for the continuity of $I_{V}$ at every $V$ is that the function $\phi[x, y, p]$ is $l i$ near with respect to each one of the vectors $p_{i}$.

REMARK 1.3. As a consequence of Theorem 1.2, the most general function $\phi[x, y, p]$, such that $\int_{D} \phi[x, y(x), p(x)] d x$ is continuous at every

1 For the relation between this integral and non local field theories see bibliography $[1,6,27,28,29,40,41,42,46,47,48,58]$. 
$V$, may be written in the form

$$
\sum_{q=1}^{m} \sum_{U_{q}} \sum_{\mu}\left\{A_{U_{q}, \mu}(x, y) \prod_{\zeta \in U_{q}} p_{\zeta}^{\mu(\zeta)}\right\},
$$

where we assume by convention that, if $\eta$ is a variable integer ranging over a set $S$ and $\left\{\alpha_{n}\right\}$ is a sequence of numbers, then

$$
\prod_{\eta \in S} \alpha_{\eta}=0 \text {, whenever } S \text { is empty . }
$$

Let $L[x, y, p, \bar{p}]$ denote a polynomial in the indeterminates

$$
\left[\bar{p}_{i}^{(j)}-p_{i}^{(j)}\right]
$$

of degree not exceeding 1 in any of the vectors $\left[\bar{p}_{i}-p_{i}\right]$, whose coefficients $W_{\sigma_{q}, \mu}(x, y, p)$ are functions of $(x, y, p)$ which are continuous together with all their derivatives of the form

$$
\frac{\partial^{q} W_{U_{q}, \mu}(x, y, p)}{\prod_{\zeta \in U_{q}} \partial x_{\zeta}^{\mu(\zeta)}},
$$

$L[x, y, p, \bar{p}]$ may be written in the form

$$
\sum_{q=1}^{m} \sum_{U_{q}} \sum_{\mu}\left\{W_{U_{q}, \mu}(x, y, p) \prod_{\zeta \in U_{q}}\left[\bar{p}_{\zeta}^{\mu(\zeta)}-p_{\zeta}^{\mu(\zeta)}\right]\right\} .
$$

Let us define the generalized Weierstrass function $E_{L}[x, y, p, \bar{p}]$ of $L_{V}$ with respect to $L[x, y, p, \bar{p}]$, by the formula

$$
E_{L}(x, y, p, \bar{p})=\phi[x, y, \bar{p}]-L[x, y, p, \bar{p}] .
$$

The integral $I_{V}=\int \phi[x, y(x), p(x)] d x$ is said to be positively quasiregular with respect to $L$ (abbreviation: $L P Q R$ ) if both the relations

$$
\begin{aligned}
& E_{L}[x, y, p, p]=0 \\
& E_{L}[x, y, p, \bar{p}] \geqq 0
\end{aligned}
$$

hold for every $x \in D$ and for every $y, p, \bar{p}$.

REMARK 1.4. Notice that if $I_{V}$ is $L P Q R$, then the element of degree 0 of the polynomial $L[x, y, p, \bar{p}]$ must be $\phi[x, y, p]$, and the vector consisting of the coefficients of the elements of degree 1 is the gradient of $[x, y, p]$ with respect to $p$ : therefore, if $m=1$, i.e., if $I_{V}$ is a usual multiple integral $[60,62]$, the fact that $I_{V}$ is $L P Q R$ completely determines the function $L[x, y, p, \bar{p}]$. This does not happen if $m>1$, as was shown by an appropriate example [30], referring to Fubini-Tonelli integrals, i.e., to the case $(m=2, n=1)$.

We say that $I_{V}$ is positively quasi-regular (abbreviation $P Q R$ ) if 
there exists at least one function $L[x, y, p, \bar{p}]$ such that $I_{V}$ is $L P Q R$.

REMARK 1.5. Let us say that $I_{V}$ is negatively quasi-regular with respect to $L$ (abbreviation: $L N Q R$ ) if $\int_{D}-\phi[x, y(x), p(x)] d x$ is $L P Q R$. Then it is easy to prove that, if $I_{V}$ is both $L_{1} P Q R$ and $L_{2} N Q R$, then $L_{1}[x, y, p, \bar{p}] \equiv L_{2}[x, y, p, \bar{p}]$, and $\phi[x, y, p]$ is a polynomial of degree not exceeding 1 in each $p_{i}$; i.e., by Theorem 1.2, $I_{V}$ is continuous. Theorem 1.2 also implies that every continuous $I_{V}$ is both $L P Q R$ and $L N Q R$ for some $L[x, y, p, \bar{p}]$.

REMARK 1.6 In the case $m=1$, our definition of positive quasiregularity reduces to the one which was given by Tonelli $[59,60]$ and Cinquini [1] for simple and multiple integrals. In this particular case, the positive quasi-regularity of an integral is equivalent to the lower convexity of its figurative, i.e., of $\phi[x, y, p]$ considered as a function of $p$ only.

In the case $n=1$, the definition of positive quasi-regularity reduces to the one given by this author for the Fubini-Tonelli integrals [30].

REMARK 1.7. If $I_{V}$ is $P Q R$, then its value is $+\infty$ at every nonordinary variety.

\section{Let us prove the following}

THeorem 2.1. If $I_{V}$ is $P Q R$, then it is lower semicontinuous at every variety $V$ of class 2; i.e., if $V$ is of class 2, there exists a positive function $\rho(\varepsilon)$ of the positive variable $\varepsilon$ such that, if $\bar{V} \equiv \bar{y}(x)$ is any variety, then

$$
I_{\bar{V}}-I_{V}>-\varepsilon, \text { whenever } \rho(V, \bar{V})<\rho(\varepsilon) .
$$

regardless of whether or not $V$ is of class 2 .

Proof. Let $L[x, y, p, \bar{p}]$ be a function, such that $l_{V}$ is $L P Q R$. By (1.6) we may write

$$
\begin{aligned}
& I_{\bar{V}}-I_{V}=\int_{D} E_{L}[x, \bar{y}(x), p(x), \bar{p}(x)] d x-\int_{D} E_{L}[x, y(x), p(x), p(x)] d x \\
& \quad+\int_{D} L[x, \bar{y}(x), p(x), \bar{p}(x)] d x-\int_{D} L[x, y(x), p(x), p(x)] d x
\end{aligned}
$$

Let $V \equiv y(x)$ be a variety of class 2 ;

$$
P[x, \bar{y}, \bar{p}] \equiv L[x, \bar{y}, p(x), \bar{p}]
$$


is a polynomial of a degree not exceeding 1 in each $\bar{p}$, and all of the derivatives

$$
\begin{aligned}
& \frac{\partial P[x, \bar{y}, \bar{p}]}{\partial \bar{p}_{r}^{s}}, \quad \frac{\partial^{2} P[x, \bar{y}, \bar{p}]}{\partial \bar{p}_{r}^{s} \partial \bar{p}_{r}^{t}}, \frac{\partial^{2 q} P[x, \bar{y}, \bar{p}]}{\prod_{1}^{g} \partial x_{\zeta}^{\mu(\zeta)} \partial \bar{p}_{\zeta}^{\mu(\zeta)}} \\
&(r=1,2, \cdots, m ; s, t=1,2, \cdots n)
\end{aligned}
$$

exist and are continuous for every $[x, \bar{y}, \bar{p}]$ and for every $q, U_{q}, \mu(\zeta)$, $r, s, t$ as a consequence of the existence and continuity of the functions (1.4) and of the partial derivatives of the first two orders of the functions $y_{r}(x),(r=1,2, \cdots, m)$.

By the continuity Theorem 1.2,

$$
J_{V}=\int_{D} P[x, \bar{y}(x), \bar{p}(x)] d x
$$

is continuous; hence the difference of the last two integrals on the right side of (2.2) is smaller than any predetermined real positive $\varepsilon$, whenever $\rho(V, \bar{V})$ is less than a certain positive number $\rho(\varepsilon)$. Since the first integral on the right side of (2.2) is not negative by (1.8) and the second vanishes by (1.7), (2.1) holds: the theorem is thus proved.

3. (a) In this section the concept of asymptotic evaluability of the integral $I_{V}$ is defined; the lower semicontinuity on every very variety $V$ of any positively quasi-regular and asymptocally evaluable integral is proved. The results of this chapter may be regarded as extensions of Tonelli's theorems on usual multiple integrals $[59,60]$, and of our results on Fubini-Tonelli integrals [30].

(b) Suppose that $I_{V}=\int_{D} \phi[x, y(x), p(x)] d x$ is $P Q R$, and let $L[x, y$, $p, \bar{p}]$ be one of the functions, such that $I_{V}$ is $L P Q R$.

The function

$$
\bar{\Phi}[x, y, p] \equiv E_{L}[x, y, \Omega, p],
$$

where $\Omega$ is a $m \cdot n$ matrix whose elements are all 0 , is never negative. Furthermore,

$$
\bar{I}_{V}=\int_{D} \bar{\Phi}[x, y(x), p(x)] d x
$$

is $\bar{L} P Q R$, where

$$
\bar{L}[x, y, p, \bar{p}]=L[x, y, p, \bar{p}]-L[x, y, \Omega, \bar{p}] .
$$

By (1.7), the equation

$$
\bar{\Phi}[x, y, \Omega]=0
$$


holds for every $x \in D$ and every $y$.

Let $R$ denote a positive real number and let $\varphi^{R}[x, y, p]$ denote a function such that the following conditions are satisfied:

I. $\varphi^{R}[x, y, \mathrm{p}]$ is continuous with all its partial derivatives of any of the forms

$$
\frac{\partial \varphi^{R}[x, y, p]}{\partial p_{r}^{s}}, \quad \frac{\partial^{2} \phi^{R}[x, y, p]}{\partial p_{r}^{s} \partial p_{r}^{t}}, \frac{\partial^{2 q} \mathcal{P}^{R}[x, y, p]}{\prod_{1}^{q} \partial x_{\zeta}^{\mu(\zeta)} \partial p_{\zeta}^{\mu(\zeta)}} .
$$

II. The integral

$$
Y_{V}=\int_{D} \varphi^{R}[x, y,(x), p(x)] d x
$$

is $P Q R$.

III. The relation

$$
0 \leqq \phi^{R}[x, y, p] \leqq \ddot{\Phi}[x, y, p]
$$

holds for every $y, p$ and for every $x \in D$; furthermore

$$
\varphi^{R}[x, y, p]=\bar{\Phi}[x, y, p], \text { whenever } \sum_{i=1}^{m} \sum_{j=1}^{n}\left(p_{1}^{j}\right)^{2} \leqq R .
$$

IV. There exists at least one function $\Lambda[x, y, p, \bar{p}]$, such that $Y_{V}$ is $\triangle P Q R$, and such that, for each $T>1$, there exists a number $Q$, for which the following condition is satisfied:

Let $q, U_{q}, \zeta, \mu(\zeta)$ be defined as they were in $\S 1$; let $\bar{U}_{q}$ denote the complement of $U_{q}$ with respect to the set of the first $m$ positive integers, and let $\bar{\zeta}$ be an index ranging over $\bar{U}_{q}$. Then the inequality

$$
\left|W_{U_{q}, \mu}^{R}[x, y, p]\right|<Q\left(1+\prod_{\bar{\zeta} \in \bar{U}_{q}} p_{\bar{\zeta}}^{\mu(\bar{\zeta})} \mid\right)
$$

where $W_{U_{q}, \mu}^{R}[x, y, p]$ denotes the coefficient of the element

$$
\prod_{\zeta \in U_{q}}\left[\bar{p}_{\zeta}^{\mu(\zeta)}-p_{\zeta}^{\mu(\zeta)}\right]
$$

of the expression $\Lambda[x, y, p, \bar{p}]$, holds for every $q, U_{q}, p$, for every $x \in D$ and for every $y$ such that

$$
\left|y_{i}\right|<T \quad(i=1,2, \cdots, n) .
$$

REMARK 3.1. In the case of the usual multiple integrals $(m=1)$, Condition IV reduces to the boundedness of the derivatives 


$$
\frac{\partial \varphi^{R}[x, y, p]}{\partial p_{1}^{s}} \quad(s=1,2, \cdots, n)
$$

in any domain where $y(x)$ is bounded; this condition is exactly the one considered by Tonelli $[59,60]$.

In the case of Fubini-Tonelli integrals $(n=1)$, this condition reduces to the one that this author considered in $[30, \S 1$, page 132].

REMARK 3.2. $Y_{V}$ exists and is finite on every variety $V$, i.e., every variety $V$ is ordinary for the integral $Y_{V}$.

(c) Lemma 3.3. The integral $Y_{V}$ defined by (3.b.4) is lower semicontinuous at every variety $V$.

Proof. Let $V \equiv y(x) \equiv\left\{y_{\imath}\left(x_{i}\right)\right\}$ be any variety; and let $1>\varepsilon>0$ and $R>0$ be given, and let $\pi \equiv \pi(x) \equiv\left\{\pi_{i}\left(x_{i}\right)\right\}$ denote a variety of class 2 , such that

$$
\rho(\pi, V)<\varepsilon
$$

Let $T=\sup _{x_{i}, i}\left|y_{i}\left(x_{i}\right)\right|+2$.

Let $\pi^{\prime}(x) \equiv\left\|\pi_{i}^{\prime j}(x)\right\| \equiv\left\|\frac{\partial \pi_{i}\left(x_{i}\right)}{\partial x_{\imath}^{j}}\right\|, \quad(i=1,2, \cdots, m ; j=1,2, \cdots, n)$,

and let $\bar{D}_{i} \subset D_{i}$ denote set of the points $x_{i}$, such that, for some $j$, either $p_{i}^{\prime}\left(x_{i}\right)$ does not exist or it is such that

$$
\left|\pi_{i}^{\prime j}\left(x_{i}\right)-p_{i}^{j}\left(x_{i}\right)\right| \geqq \varepsilon .
$$

Suppose further that, for each $i(i=1,2, \cdots, m)$,

$$
\int_{\bar{D}_{i}} \sum_{1}^{n}\left[\left|\pi_{i}^{\prime j}\left(x_{i}\right)\right|+\left|p_{i}^{j}\left(x_{i}\right)\right|\right] d x_{i}<\varepsilon .
$$

The construction of such a variety $\pi$ is possible for any $V$ [68]. If $\bar{V}=\bar{y}(x) \equiv\left\{\bar{y}_{i}(x)\right\}$ is any other variety, we may write

$$
\begin{aligned}
Y_{\bar{V}} & -Y_{V}=\int_{D} E_{A}^{\varphi}\left[x, \bar{y}(x), \pi^{\prime}(x), \bar{p}(x)\right] d x \\
& -\int_{D} E_{\Lambda}^{\varphi}\left[x, y(x), \pi^{\prime}(x), p(x)\right] d x \\
& +\int_{D} \Lambda\left[x, \bar{y}(x), \pi^{\prime}(x), \bar{p}(x)\right] d x \\
& -\int_{D} \Lambda\left[x, y(x), \pi^{\prime}(x), p(x)\right] d x
\end{aligned}
$$

where

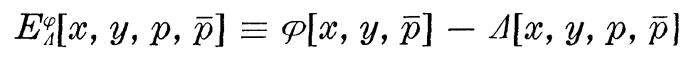


The first integral on the right side of (3.c.4) may not be negative because $Y_{V}$ is $P Q R$; since $\pi$ is a variety of class 2 , we may show in the same way as we did for proving Theorem 2.1, that there exists a $0<\rho_{1}<1$, such that, if $P(V, \bar{V})<\rho_{1}$, then the difference between the last two integrals on the right side of (3.c.4) is less than $\varepsilon$.

Let us consider the expression

$$
\int_{D}\left|E_{A}^{\varphi}\left[x, y(x), \pi^{\prime}(x), p(x)\right]\right| d x
$$

by (3.c.5), (3.b.7) and (3.c.3), recalling the defininition of $A[x, y, p, \bar{p}]$, i.e., the definition of $L[x, y, p, \bar{p}]$, since $\pi^{\prime}\left(x_{i}\right)(i=1,2, \cdots, m)$ is bounded, there exists a number $k$, which depends upon $m, n$, the variety $V$ and the diameters of the sets $D_{i}(i=1,2, \cdots, m)$, but which depends neither of $\pi$ nor of $\varepsilon$, such that the expression (3.c.6) is less than $\varepsilon \cdot k$ ([59, vol. $1, \S 11, \# 142] ;[60, \S 3, \# 9] ;[30, \S 3, \mathrm{c}])$. Consequently the absolute value of the integral on the right side of (3.c.4) is also less than $\varepsilon \cdot k$; hence

$$
Y_{\bar{V}}>Y_{V}-\varepsilon(1+k) \text {, whenever } \rho(V, \bar{V})<\rho_{1} .
$$

Thus the theorem is proved.

(d) Definition 3.4. Then integral $I_{V}$ is said to be asymptotically evaluable (abbreviation: $A E$ ), if it is $P Q R$ and if there exists a function $L[x, y, p, \bar{p}]$ such that $I_{V}$ is $L P Q R$ and if, for every positive $R$, there exists a function $\varphi^{R}[x, y, p]$ (as described in $\S 3 . b$ ).

REMARK 3.5. Tonelli [59, vol. 1, page 398-9] gave a procedure by which $\varphi^{R}[x, y, p]$ may be constructed starting from any simple integral ( $m=n=1)$, which is $P Q R$ : he thus proved that, if a simple integral is $P Q R$, it is necessarily $A E$. Some criteria of asymptotic evaluability are exhibited in [30, $\S 2$, page 140]; although it appears intuitively that every $P Q R$ integral is also $A E$, this fact was never proved, except in the case $(m=n=1)$; therefore the statement of any theorem of semicontinuity in whose proof the function $\varphi_{R}[x, y, p]$ is used, has to contain the hypothesis that this function can be constructed, i.e., that the integral considered is $A E$.

THEOREM 3.6. If the integral $I_{V}$ is $P Q R$ and $A E$, it is lower semicontinuous on every ordinary variety.

Proof. Let us first point out that existence and lowers emicontinuity on any variety of $I_{V}$, and those of the integral $\bar{I}_{V}$ defined by (3.b.2), are equivalent, since the integral 


$$
\int_{D}\{\Phi[x, y(x), p(x)]-\bar{\Phi}[x, y(x), p(x)]\} d x \equiv \int_{D} L[x, y(x), \Omega, p(x)] d x
$$

exists and is continuous at every variety, by the Continuity Theorem 1.2 .

Let $V=y(x) \equiv y_{i}\left(x_{i}\right)$ be an ordinary variety, and let $\varepsilon>0$ be given.

Since $\bar{\Phi}[x, y, p]$ is never negative, it is possible to find a positive number $R$, such that, if $D_{i}^{\prime}(i=1,2, \cdots, m)$ is the subset of $D_{i}$ consisting of the points $x_{i}$ such that, for each $j(j=1,2, \cdots, n)$, the partial derivative $\partial y_{i}\left(x_{i}\right) / \partial x_{i}^{j}$ exists and its absolute value does not exceed $R$, the inequality

$$
\bar{I}_{V}-\int_{I_{1} i D_{\imath}^{\prime}}^{m} \bar{\Phi}[x, y(x), p(x)] d x<\varepsilon / 2
$$

holds.

The integral $Y_{V}$, that we associate with $I_{V}$ and $R$ (see $\S 3 . b$ ) is lower semicontinuous on $V$ by Lemma 3.3; i.e., there exists a positive number $\bar{\rho}$ such that, for each variety $\bar{V}$

$$
Y_{\bar{V}}>Y_{V}-\varepsilon / 2 \text {, whenever } \rho(V, \bar{V})>\bar{\rho} .
$$

From (3.b.5) and (3.d.1) we have

$$
\bar{I}_{V}-Y_{V}<\varepsilon / 2
$$

whence, by (3.d.2)

$$
\bar{I}_{\bar{V}}>\bar{I}_{V}-\varepsilon, \text { whenever } \rho(V, \bar{V})<\bar{\rho}
$$

i.e., $\bar{I}_{V}$ is lower semicontinuous at any ordinary variety, and so is $I_{V}$.

Definition 3.7. We shall say that the integral $I_{V}$ is lower semicontinuous at a variety $V$, such that $I_{V}=+\infty$, if there exists a positive function $\rho(\varepsilon)$, defined for each positive $\varepsilon$, such that, if $\bar{V}$ is any ordinary variety, then

$$
I_{\bar{V}}>\varepsilon, \text { whenever } \rho(V, \bar{V})<\rho(\varepsilon) \text {. }
$$

THeOREm 3.8. An integral $I_{V}$, which is $P Q R$ and $A E$, is lower semicontinuous at every variety $V$.

In the case in which $V$ is ordinary, Theorem 3.6 states the lower semicontinuity of $I_{V}$ on $V$. If $V$ is not ordinary, the value of $I_{V}$ on $V$ is $+\infty$ (see Remark 1.7).

Let us again consider $\bar{I}_{V}$ instead of $I_{V}$. Let $\varepsilon$ be a given number, and let $R$ be another positive number, such that, if $\overline{\bar{D}}_{i}(i=1,2, \cdots$, $m$ ) denotes the subset of $D_{i}$ consisting of the points $x_{i}$ where all the 
partial derivatives of $y_{i}\left(x_{i}\right)$ exist and are less than $R$ in absolute value, then

$$
\left.\int_{\substack{\Pi_{i} \\ i_{i}}} \overline{\bar{D}_{i}} \underset{\Phi}{ } \bar{\Phi}, y(x), p(x)\right] d x>\varepsilon+1 .
$$

Like in the proof of Theorem 3.6, we consider again $\phi^{R}[x, y, p]$. $Y_{V}$ exists finite and is lower semicontinuous at $V$ : hence we may find a positive number $\bar{\rho}$, such that, if $V$ is any variety such that

$$
\rho(V, \bar{V})<\bar{\rho},
$$

then

$$
Y_{\bar{V}}>Y_{\bar{V}}-1
$$

By (3.b.5) and (3.b.6),

$$
\begin{aligned}
Y_{V} & \geqq \int_{\prod_{I_{i}}^{m} \overline{\bar{D}}_{i}} \mathcal{P}^{R}[x, y(x), p(x)] d x \\
& =\int_{\prod_{i} i \overline{\bar{D}}_{i}}^{m} \bar{\Phi}[x, y(x), p(x)] d x,
\end{aligned}
$$

hence, considering (3.e.1), if (3.e.2) is satisfied,

$$
\bar{I}_{\bar{V}}>\varepsilon \text {. }
$$

Therefore $\bar{I}_{V}$ is semicontinuous at $V$, and so is $I_{V}$. The theorem is thus completely proved.

Conclusion. Let us list four problems which are still open in the area of the study of the semicontinuity of the integrals of the Calculus. of Variations in non-parametric form:

Problem 1. No example of any lower semicontinuous integral which is not $P Q R$ is known: it appears worth while to investigate whether or not positive quasi-regularity is also necessary for lower semicontinuity.

Problem 2. For proving Theorems 3.6,3.8, we used the construction of the function $\varphi^{R}[x, y, p]$, and we had to assume that this construction could be made for every $R$ (see $\S 3 . b$ ). It would be interesting to prove Theorem 3.8 without using this construction, i.e., dropping the hypothesis that $I_{V}$ is $A E$.

REMARK C.1. The semicontinuity at any variety $V$ of class 1 , or even just such that all the functions $y_{i}\left(x_{i}\right)$ are Lipschitzian, can easily be proved for any $I_{V}$, which is $P Q R$, without any hypothesis of asymp- 
totic evaluability, by generalizing the procedure followed in $[30, \S 3$, First Theorem of Semicontinuity].

Problem 3. No example of any integral $I_{V}$, which is $P Q R$ without being $A E$, is known. It would be useful to devise a general method by which it would be possible to construct $\mathcal{P}^{R}[x, y, p]$ from $R$ and $\phi[x, y, p]$ : thus proving that if $I_{V}$ is $P Q R$, it is necessarily $A E$.

Problem 4. Only varieties which are absolutely continuous in the sense of Tonelli [63] and the m-uniform metric were considered in this paper; however, it appears that positively quasi-regular integrals are lower semicontinuous even with respect to weaker metrics, on more general classes of varieties. Generalization of the results contained in this paper may be considered.

\section{BIBLIOGRAPHY}

1. C. Bloch, On field theories with non-localized interactions, Det Kongelige Danske Videnskabernes Selskab, Matematisk-fysiske Meddeleser, (1952).

2. S. Cinquini, Condizioni necessarie per la semicontinuitá degli integrali doppi del Calcolo delle Variazioni, Annali Matem. Pura ed Appl., 10 (1932), 233.

3. Nuovi teoremi di esistenza dell'estremo in campi illimitati per $i$ problemi di ordine n, Ann. Scuola Norm. Super. Pisa, (1930).

$4 . \quad$ L L'estremo assoluto degli integrali doppi dipendenti dalle derivate di ordine superiore, Ann. Scuola Norm. Super. Pisa (2) 10 (1941), 215.

5. S__ Sopra l'estremo assoluto degli integrali doppi in forma ordinaria, Ann. Mat. Pura Appl. 30 (1949), 249.

6. M. E. Ebel, Casual Behaviour of Field Theories with Non-Localized Interactions, (Magazine quoted in [1] , 1954).

7. S. Faedo, Condizioni necessarie per la semicontinuitá di un nuovo tipo di funzionali, Ann. Mat. Pura Appl. (4) 23 (1944), 69.

8. __ Un nuovo tipo di funzionali continui, Rend. Mat. Appl. (5) 4 (1943), 1.

9 . Sulle condizioni di Legendre e di Weierstrass per gli integrali di Fubini-

Tonelli, Ann. Scuola Norm. Super. 15 (1950), 127.

10. L. Giuliano, Sulle condizioni sufficienti per la semicontinuitá degli integrali doppi del Calcolo delle Variazioni, Ann. Scuola Norm. Super. Pisa (3) 1 (1941), 161.

11. __ Osservazioni sopra alcuni teoremi di semicontinuitá degli integrali doppi (Idem, 1941).

12. La variazione prima nei problemi di Lagrange, (Rend. di Mat. App. (1947).

13. Sulla continuitá degli integrali curvilinei del Calcolo delle Variazioni (Nota I), (Rend. dell'Acc. Naz. Lincei, (1947).

14. _ Idem (Nota II), (Idem, 1947).

$15 . \quad$ Idem (Nota III), (Idem, 1949).

16. Sulla continuitó degli integrali curvilinei del Calcolo delle Variazioni, (Ann. della Scuola Normale Superiore, Pisa, (1947).

17. M. R. Hestenes, A sufficiency Proof for Isoperimetric Problems in the Calculus of Variations, Bull. Am. Math. Soc. (1938).

18. Sufficient Conditions for Multiple Integral Problems in the Calculus of Variations Amer. J. Math. 70 (1948), 239. 
19. Calculus of variations, Pacific J. Math. 1 (1951), 525.

20. Sufficient Conditons for the General Problem of Mayer with Variable End. Points, University of Chicago Press, (1952).

21. On sufficient conditions in the problems of Langrange and Bolza, Ann. Math. 37 (1936), 543.

22. _ A Direct Sufficiency proof for the Problem of Bolza in the Calculus of Variations, Trans. Amer. Math. Soc. 42 (1947), 141.

$23 . \quad$ Generalized problem of Bolza in the calculus of variations, Duke Math. J. 5 (1939), 309.

24. The problem of Bolza in the Calculus of Variations, Bull. Amer. Math. Soc. 48 (1942), 50.

25. An Alternate Sufficiency Proof for the Normal Problem of Bolza, Trans. Amer. Math. Soc. 61 (1947), 256.

26. _ An indirect Sufficiency Proof for the Normal Problem of Bolza in NonParametric Form Trans. Amer. Math. Soc. 62 (1947), 509.

27. P. Kristensen, and C. Moller, On a Convergent Meson Theory, Journal quoted in [1], (1952). 28. A. Kryzwiki, I. Rzewuski, I. Zamorski, and A. Zieba, Non local problems in the Calculus of Variations, Annales Polonici Matematici, (1955).

29. A. Krzywiki, I. Rzewuski, I. Zamorski, and A. Zieba, Idem, Second Paper, Idem, (1957) 30. L. Lombardi, Sulla semicontinuitá degli integrali di Fubini-Tonelli, Annali della Scuola Normale Superiore, Pisa 312 (1958), 129-153.

31. _ Sull'esistenza del minimo degli integrali di Fubini-Tonelli, Rendiconti dell'Istituto Lombardo Sci. A 92 (1958), 446-458.

32. Sur la Méthode du Gradient dans le Calcul des Variations, Comptes-Rendus du Congrés Les Mathématiques de l'Ingénieur, 216-225 Mons-Bruxelles, (1958).

33. The continuity of the most general integral of the calculus of variations in non-parametric form, J. of Mathematical Analysis and Applications (in press).

34. E. Magenes, Intorno agli integrali di Fubini-Tonelli: condizioni sufficienti per la semicontinuitá, Annali della Scuola Normale Superiore, Pisa (3) 2 (1948), 1.

35. _ Intorno agli integrali di Fubini-Tonelli: teoremi di esistenza dell'estremo, (Idem, (3) 3 65, 1949).

36. __ Sul minimo degli integrali di Fubini-Tonelli, Giornale di Battaglini, Napoli, (1949-50).

37. , Sulle condizioni di Eulero relative agli integrali di Fubini-Tonelli, Rend. Sem. Math. Padova. 19 (1950), 62.

38. , Un'osservaione sulle condizioni necessarie per la semicontinuitá degli in. tegrali di Fubini-Tonelli, (Idem, 17 44, 1950).

39. _ Sul minimo semiforte degli integrali di Fubini-Tonelli, (Idem, 20401 1950). 40. C. Moller, Non-local Field Theory (Intern. Conference on Elementary Particles, Bornbay, (1950).

41. __ On the Problem of Convergence in Non-local Field Theories, Proc.;of the Intern. Congress on Theoret. Physics, Kyoto and Tokyo, (1953).

42. Motion of free particles in discontinuous gravitational fields, Amer. J. of Physics, (1959).

43. C.B. Morrey, Functions of several variables and absolute continuity, Duke Math, J. 6 (1940), 187.

44. , Multiple Integral Problems in the Calculus of Variations and Related Topics, University of California Publ. in Math, (1943).

45. ( Quasi-convexity and the lower semicontinuity of multiple integrals and related topics, Pacific. J. Math. 2 (1952).

46. W. Pauli, On the Hamiltonian structure of Non-local field theories, Nuovo Cimento, 10 (1953), 648.

47. I. Rzewuski, On differential structure of non-local field theories, Bull. de l'Academie 
Polonaise des Sciences, 2 (1954), 429.

48. , differential structure of non-local theories, Acta Physica Polonica, 14 (1955),

121.

49. B. Schweizer, A. Sklar, Statistical metric spaces, Pacific. J. Math. 1 (1960).

50. , Statistical metric spaces arising from sets of random variables in Euclidean $n$-space, (Forthcoming).

51. G. I. Silova, The existence of the absolute minimum of the multiple integrals of the calculus of variations, Doklady Akad. Nauk SSSR, 102 (1955), 669.

52. _. Two dimensional problems of the calculus of variations, Uspechi Akad. Nauk SSSR, 6 (1951), 16.

53. A. G. Sigalov, Conditions for the existence of a minimum of double integrals in an unbounded region, Doklady Ak. Nauk SSSR, 8 (1951), 741.

54. , Regular Double Integrals of the Calculus of Variations in Non-Parametric Form, (Idem, 73 891, 1950).

55. _ Two dimensional problems of the calculus of variations transformed from non-parametric to parametric form, Mat. Sbornik, 73 (1954), 385.

56. G. Stampacchia, Sopra una classe di funzioni di due variabili; applicazioni agli integrali doppi del Calcolo delle Variazioni, Giorn. di Battaglini, 79 (1950), 169.

57. _ Gli integrali doppi del Calcolo delle Variazioni in forma ordinaria, Atti Accad. Naz. dei Lincei, VIII 8 (1950), 21.

58. E. C. G. Stueckelberg, G. Wanders, Acausalité de l'intéraction non-locale, Helv. Phys. Acta, 27 (1954), 667.

59. L. Tonelli, Fondamenti di Calcolo delle Variazioni, Zanichelli, Bologna, 1921, vol. 1 and 2).

60. , Sur la semicontinuité des intégrales doubles du calcul des variations, Acta Math., 53 (1939), 323.

61. Gli integrali del Calcolo delle Variazioni in forma ordinaria, Annali della Scuola Superiore, Pisa, (2) 3 (1934), 401.

62. L_ L'estremo assoluto degli integrali doppi, (Idem, (2) 2 89, 1933).

63. — Sull'approssimazione analitica delle funzioni di piú variabili, Rend. Circ.

Mat. Palermo, 32 (1910), 297.

64. — Sui massimi e minimi assoluti del Calcolo delle variazioni, (Idem, 1911).

65. _ - Sul caso regolare nel calcolo delle variazioni, (Idem, $\mathbf{3 5}$ 49, 1913).

66. ___ Sur une méthode directe du Calcul des Variations, (Idem, 39 233, 1915).

67. __ La semicontinuitá nel Calcolo delle Variazioni, (Idem, 44 167, 1920).

68. __ Sopra alcune proprietá di un polinomio di approssimazione, Rend. Acc.

Naz. dei Lincei, (1926).

UNiversity of CALIFornia, Los ANGeles 



\section{PACIFIC JOURNAL OF MATHEMATICS}

\section{EDITORS}

\author{
RaLPh S. Phillips \\ Stanford University \\ Stanford, California \\ F. H. BRowNELL \\ University of Washington \\ Seattle 5 , Washington
}

A. L. Whiteman

University of Southern California

Los Angeles 7, California

L. J. Paige

University of California

Los Angeles 24, California

\author{
E. F. BECKENBACH \\ T. M. CHERRY
}

\author{
ASSOCIATE EDITORS

$\begin{array}{lll}\text { D. DERRY } & \text { H. L. ROYDEN } & \text { E. G. STRAUS } \\ \text { M. OHTSUKA } & \text { E. SPANIER } & \text { F. WOLF }\end{array}$

\section{SUPPORTING INSTITUTIONS}

\author{
UNIVERSITY OF BRITISH COLUMBIA \\ CALIFORNIA INSTITUTE OF TECHNOLOGY \\ UNIVERSITY OF CALIFORNIA \\ MONTANA STATE UNIVERSITY \\ UNIVERSITY OF NEVADA \\ NEW MEXICO STATE UNIVERSITY \\ OREGON STATE COLLEGE \\ UNIVERSITY OF OREGON \\ OSAKA UNIVERSITY \\ UNIVERSITY OF SOUTHERN CALIFORNIA
}

\author{
STANFORD UNIVERSITY \\ UNIVERSITY OF TOKYO \\ UNIVERSITY OF UTAH \\ WASHINGTON STATE COLLEGE \\ UNIVERSITY OF WASHINGTON \\ AMERICAN MATHEMATICAL SOCIETY \\ CALIFORNIA RESEARCH CORPORATION \\ HUGHES AIRCRAFT COMPANY \\ SPACE TECHNOLOGY LABORATORIES \\ NAVAL ORDNANCE TEST STATION
}

Mathematical papers intended for publication in the Pacific Journal of Mathematics should be typewritten (double spaced), and the author should keep a complete copy. Manuscripts may be sent to any one of the four editors. All other communications to the editors should be addressed to the managing editor, L. J. Paige at the University of California, Los Angeles 24, California.

50 reprints per author of each article are furnished free of charge; additional copies may be obtained at cost in multiples of 50 .

The Pacific Journal of Mathematics is published quarterly, in March, June, September, and December. The price per volume (4 numbers) is $\$ 12.00$; single issues, $\$ 3.50$. Back numbers are available. Special price to individual faculty members of supporting institutions and to individual members of the American Mathematical Society: $\$ 4.00$ per volume; single issues, $\$ 1.25$.

Subscriptions, orders for back numbers, and changes of address should be sent to Pacific Journal of Mathematics, 103 Highland Boulevard, Berkeley 8, California.

Printed at Kokusai Bunken Insatsusha (International Academic Printing Co., Ltd.), No. 6, 2-chome, Fujimi-cho, Chiyoda-ku, Tokyo, Japan.

\section{PUBLISHED BY PACIFIC JOURNAL OF MATHEMATICS, A NON-PROFIT CORPORATION}

The Supporting Institutions listed above contribute to the cost of publication of this Journal, but they are not owners or publishers and have no responsibility for its content or policies.

Reprinted 1966 in the United States of America 


\section{Pacific Journal of Mathematics}

\section{Vol. 11, No. 4}

A. V. Balakrishnan, Prediction theory for Markoff processes . . . . . . . . . . 1171

Dallas O. Banks, Upper bounds for the eigenvalues of some vibrating systems . . . . 1183

A. Białynicki-Birula, On the field of rational functions of algebraic groups ...... 1205

Thomas Andrew Brown, Simple paths on convex polyhedra .............. 1211

L. Carlitz, Some congruences for the Bell polynomials . . . . . . . . . . . . 1215

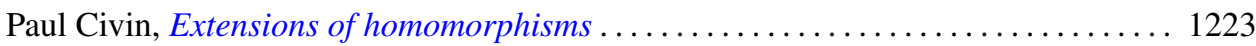

Paul Joseph Cohen and Milton Lees, Asymptotic decay of solutions of differential

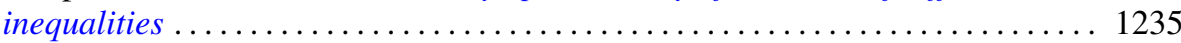

István Fáry, Self-intersection of a sphere on a complex quadric . . . . . . . . . . 1251

Walter Feit and John Griggs Thompson, Groups which have a faithful representation

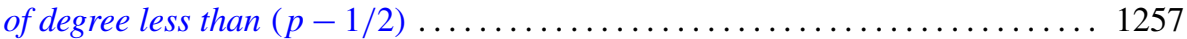

William James Firey, Mean cross-section measures of harmonic means of convex

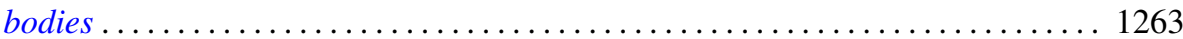

Avner Friedman, The wave equation for differential forms . . . . . . . . . . 1267

Bernard Russel Gelbaum and Jesus Gil De Lamadrid, Bases of tensor products of

Banach spaces ................................... 1281

Ronald Kay Getoor, Infinitely divisible probabilities on the hyperbolic plane . . . . 1287

Basil Gordon, Sequences in groups with distinct partial products . . . . . . . . . . . . 1309

Magnus R. Hestenes, Relative self-adjoint operators in Hilbert space . . . . . . . . . 1315

Fu Cheng Hsiang, On a theorem of Fejér ......................... 1359

John McCormick Irwin and Elbert A. Walker, On N-high subgroups of Abelian

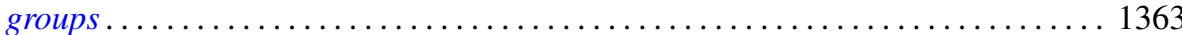

John McCormick Irwin, High subgroups of Abelian torsion groups . . . . . . . . . 1375

R. E. Johnson, Quotient rings of rings with zero singular ideal . . . . . . . . . . . 1385

David G. Kendall and John Leonard Mott, The asymptotic distribution of the time-to-escape for comets strongly bound to the solar system ...

Kurt Kreith, The spectrum of singular self-adjoint elliptic operators ....

Lionello Lombardi, The semicontinuity of the most general integral of the calculus of variations in non-parametric form ................................

Albert W. Marshall and Ingram Olkin, Game theoretic proof that Chebyshev inequalities are sharp

Wallace Smith Martindale, III, Primitive algebras with involution . . William H. Mills, Decomposition of holomorphs ..............

James Donald Monk, On the representation theory for cylindric algebras . . . . . . 1447

Shu-Teh Chen Moy, A note on generalizations of Shannon-McMillan theorem . . . . 1459

Donald Earl Myers, An imbedding space for Schwartz distributions . .

John R. Myhill, Category methods in recursion theory .........

Paul Adrian Nickel, On extremal properties for annular radial and circular slit mappings of bordered Riemann surfaces

Edward Scott O'Keefe, Primal clusters of two-element algebras . .

Nelson Onuchic, Applications of the topological method of Wazewski to certain

problems of asymptotic behavior in ordinary differential equations ...

Peter Perkins, A theorem on regular matrices................

Clinton M. Petty, Centroid surfaces .... 